\title{
Search for lepton-flavour-violating decays of Higgs-like bosons
}

\section{LHCb Collaboration ${ }^{\star}$}

CERN, 1211 Geneva 23, Switzerland

Received: 23 August 2018 / Accepted: 29 October 2018 / Published online: 12 December 2018

(C) CERN for the benefit of the LHCb collaboration 2018

\begin{abstract}
A search is presented for a Higgs-like boson with mass in the range 45 to $195 \mathrm{GeV} / c^{2}$ decaying into a muon and a tau lepton. The dataset consists of proton-proton interactions at a centre-of-mass energy of $8 \mathrm{TeV}$, collected by the $\mathrm{LHCb}$ experiment, corresponding to an integrated luminosity of $2 \mathrm{fb}^{-1}$. The tau leptons are reconstructed in both leptonic and hadronic decay channels. An upper limit on the production cross-section multiplied by the branching fraction at $95 \%$ confidence level is set and ranges from $22 \mathrm{pb}$ for a boson mass of $45 \mathrm{GeV} / c^{2}$ to $4 \mathrm{pb}$ for a mass of $195 \mathrm{GeV} / c^{2}$.
\end{abstract}

\section{Introduction}

Decays mediated by charged-lepton flavour-violating (CLFV) processes are forbidden in the Standard Model (SM). Their observation would be a clear sign for physics beyond the SM. Such processes are predicted by several theoretical models [1-8], in particular those based on an effective theory with relaxed renormalisability requirements [9], supersymmetric models [10-14], composite Higgs models [15,16], RandallSundrum models $[17,18]$, and non-abelian flavour symmetry models [19]. Nonetheless, no evidence for CLFV effects has been reported to date.

The LEP experiments set stringent limits on the CLFV decay of the $Z$ boson [20-23]. In the presence of CLFV couplings, the decays to $e^{ \pm} \mu^{\mp}, e^{ \pm} \tau^{\mp}$ and $\mu^{\mp} \tau^{\mp}$ could be mediated by a Higgs boson. At LEP2, limits on the cross-section of the $e^{+} e^{-} \rightarrow e^{ \pm} \mu^{\mp}, e^{+} e^{-} \rightarrow e^{ \pm} \tau^{\mp}$ and $e^{+} e^{-} \rightarrow \mu^{ \pm} \tau^{\mp}$ processes were obtained by the OPAL collaboration for centre-of-mass energies $(\sqrt{s})$ ranging from 192 to $209 \mathrm{GeV}$ [24]. These constraints can be translated into limits on the Higgs CLFV decay branching fraction [9,25], which are on the order of $10^{-8}$ for a SM Higgs decay into an electron and muon [25]. Recent searches for the $H \rightarrow \mu^{ \pm} \tau^{\mp}$ decay have been performed by the CMS [26] and ATLAS [27] collaborations for the Higgs boson with $m_{H}=125 \mathrm{GeV} / c^{2}$. Upper limits on the branching fraction $\mathcal{B}\left(H \rightarrow \mu^{ \pm} \tau^{\mp}\right)$ have

^e-mail: chitsanu.khurewathanakul@epfl.ch been placed by the two collaborations at $0.25 \%$ and $1.85 \%$, respectively.

The possible existence of low-mass Higgs-like bosons is a feature of models like the two-Higgs-doublet models (2HDM) [28]. Searches for such particles have been performed by the ATLAS [29] and CMS [30] collaborations in the ditau decay mode. Another scenario is that of a hidden gauge sector [31,32]. In this context, the BaBar and Belle collaborations have performed searches for a resonance with a mass below $10 \mathrm{GeV} / c^{2}[33,34]$. The LHCb collaboration has recently published the results of a search for dark photons decaying into the dimuon channel, placing a stringent limit for the production of a dimuon in the mass range from 10.6 to $70 \mathrm{GeV} / c^{2}$ [35].

The $\mathrm{LHCb}$ detector probes the forward rapidity region which is only partially covered by the other LHC experiments, and triggers on particles with low transverse momenta $\left(p_{\mathrm{T}}\right)$, allowing the experiment to explore relatively small boson masses. In this paper a search for CLFV decays into a muon and a tau lepton of a Higgs-like boson with a mass ranging from 45 to $195 \mathrm{GeV} / c^{2}$ is presented, using proton-proton collision data collected at $\sqrt{s}=8 \mathrm{TeV}$. The Higgs-like boson is assumed to be produced by gluon-fusion, similarly to the main production mechanism of the SM Higgs boson at LHC [36]. ${ }^{1}$ The analysis is separated into four channels depending on the final state of the $\tau$ lepton decay: (i) single muon $\tau^{-} \rightarrow \mu^{-} \bar{v}_{\mu} \nu_{\tau}$, (ii) single electron $\tau^{-} \rightarrow e^{-} \bar{v}_{e} \nu_{\tau}$, (iii) single charged hadron $\tau^{-} \rightarrow \pi^{-}\left(\pi^{0}\right) \nu_{\tau}$, and (iv) three charged hadrons $\tau^{-} \rightarrow \pi^{-} \pi^{-} \pi^{+}\left(\pi^{0}\right) \nu_{\tau}$. They are denoted as $\tau_{\mu}, \tau_{e}, \tau_{h 1}$, and $\tau_{h 3}$ respectively. The main sources of background are $Z \rightarrow \tau^{+} \tau^{-}$decays, ${ }^{2}$ heavy flavour production from QCD processes ("QCD" in the following) and electroweak boson production accompanied by jets (" $V j$ "). This analysis utilizes reconstruction techniques and results

\footnotetext{
${ }^{1}$ The remaining Higgs production modes (e.g., $\sim 10 \%$ from VectorBoson Fusion) are neglected in this study.

2 Throughout this note, $Z$ implies $Z / \gamma^{*}$, i.e. includes contributions from $Z$ boson production, virtual photon production, and also their interference.
} 
obtained from the $Z \rightarrow \tau^{+} \tau^{-}$measurement by the $\mathrm{LHCb}$ collaboration [37].

\section{Detector and simulation description}

The $\mathrm{LHCb}$ detector $[38,39]$ is a single-arm forward spectrometer covering the $2<\eta<5$ pseudorapidity range, designed for the study of particles containing $b$ or $c$ quarks. The detector includes a high-precision tracking system consisting of a silicon-strip vertex detector surrounding the $p p$ interaction region, a large-area silicon-strip detector located upstream of a dipole magnet with a bending power of $4 \mathrm{Tm}$, and three stations of silicon-strip detectors and straw drift tubes placed downstream of the magnet. The tracking system provides a measurement of the momentum of charged particles with a relative uncertainty that varies from $0.5 \%$ at low momentum to $1.0 \%$ at $200 \mathrm{GeV} / c$. The minimum distance of a track to a primary vertex (PV), the impact parameter (IP), is measured with a resolution of $\left(15+29 / p_{\mathrm{T}}\right) \mu \mathrm{m}$, where $p_{\mathrm{T}}$ is the component of the momentum transverse to the beam, in $\mathrm{GeV} / c$. Photons, electrons and hadrons are identified by a calorimeter system consisting of scintillating-pad (SPD) and preshower detectors (PS), an electromagnetic calorimeter (ECAL) and a hadronic calorimeter (HCAL). Muons are identified by a system composed of five stations of alternating layers of iron and multiwire proportional chambers.

Simulated data samples are used to calculate the efficiency for selecting signal processes, to estimate the residual background level, and to produce templates for the fit used to determine the signal yield. For this analysis, the simulation is validated primarily by comparing $Z \rightarrow l^{+} l^{-}$decays in simulation and data. The Higgs boson is generated assuming a gluon-fusion process, and with mass values from 45 to $195 \mathrm{GeV} / c^{2}$ in steps of $10 \mathrm{GeV} / c^{2}$, using Pythia 8 [40,41] with a specific LHCb configuration [42]. The parton density functions (PDF) are taken from the CTEQ6L set [43]. Decays of hadronic particles are described by EvTGEN [44], in which final-state radiation is generated using Рнотоs [45]. The interaction of the particles with the detector and its response are implemented using the GEANT4 toolkit [46,47] as described in Ref. [48]. Samples of $H \rightarrow \mu^{ \pm} \tau^{\mp}$ decays generated at next-to-leading order precision by POWHEGBox [49-52] with the PDF set MMHT2014nlo68cl [53] are used for the signal acceptance determination.

\section{Signal selection}

This analysis uses data corresponding to a total integrated luminosity of $1976 \pm 23 \mathrm{pb}^{-1}$ [54]. The data collected uses a trigger system consisting of a hardware stage followed by a software stage. The hardware trigger requires a muon track identified by matching hits in the muon stations, as well as a global event cut (GEC) requiring the hit multiplicity in the SPD to be less than 600 . The software trigger selects muons or electrons with a minimum $p_{\mathrm{T}}$ of $15 \mathrm{GeV} / c$.

The $H \rightarrow \mu^{ \pm} \tau^{\mp}$ candidates are identified and reconstructed into the four channels: $\mu \tau_{e}, \mu \tau_{h 1}, \mu \tau_{h 3}$ and $\mu \tau_{\mu}$. The $\tau_{h 3}$ candidates are reconstructed from the combination of three charged hadrons from a secondary vertex (SV). The $\mu^{ \pm} \tau^{\mp}$ candidates are required to be compatible with originating from a common PV. The muon track and the tracks used to reconstruct the tau candidate must be in the geometrical region $2.0<\eta<4$.5. Electron candidates are chosen amongst tracks failing the muon identification criteria and falling into the acceptance of the PS, ECAL, and HCAL sub-detectors. A large energy deposit, $E$, in the PS, ECAL, but not in HCAL is required, satisfying: $E_{\mathrm{PS}}>50 \mathrm{MeV}$, $E_{\mathrm{ECAL}} / p>0.1$, and $E_{\mathrm{HCAL}} / p<0.05$, where $p$ is the reconstructed momentum of the electron candidate, after recovering the energy of the bremsstrahlung photons [55]. Charged hadrons are required to be in the HCAL acceptance, to deposit an energy $E_{\mathrm{HCAL}}$ with $E_{\mathrm{HCAL}} / p>0.05$, and to fail the muon identification criteria. The pion mass is assigned to all charged hadrons.

The selection criteria need to be optimised over the $m_{H}$ range used in this analysis, from 45 to $195 \mathrm{GeV} / c^{2}$. Three different sets of selection criteria are considered, dubbed $\mathrm{L}$-selection, $\mathrm{C}$-selection, and $\mathrm{H}$-selection. The $\mathrm{C}$-selection is similar to that used for the analysis of $Z \rightarrow \tau^{+} \tau^{-}$ decays [37]; as such, it is optimised for $m_{H} \sim m_{Z}$. The Lselection and $\mathrm{H}$-selection are optimised for the $m_{H}$ regions below and above the $Z$ mass respectively. All selection sets are applied in parallel to compute background estimation and exclusion limits. Subsequently, for each $m_{H}$ hypothesis, the chosen selection is that of L-, C-, or H-selection which provides the smallest expected signal limit, allowing precise separation between adjacent mass regions. As expected, it is found that the C-selection is optimal for a boson mass of 75 and $85 \mathrm{GeV} / c^{2}$. Below and above that range the best upper limits are obtained from the $\mathrm{L}$ - and $\mathrm{H}$-selections, respectively. In the following discussion the requirements are applied identically for all decay channels and selection sets unless stated otherwise.

The tau candidates are selected with $p_{\mathrm{T}}>5 \mathrm{GeV} / c$ for $\tau_{e}, \tau_{\mu}$, and $p_{\mathrm{T}}>10 \mathrm{GeV} / c$ for $\tau_{h 1}$. For the $\tau_{h 3}$ candidate, the charged hadrons are required to have $p_{\mathrm{T}}>1 \mathrm{GeV} / c$ and one of them with $p_{\mathrm{T}}>6 \mathrm{GeV} / c$. They are combined to form the tau candidates, which are required to have $p_{\mathrm{T}}>12 \mathrm{GeV} / c$ and an invariant mass in the range 0.7 to $1.5 \mathrm{GeV} / c^{2}$. In the $\mathrm{H}$-selection, the tau candidates must have $p_{\mathrm{T}}$ in excess of $20 \mathrm{GeV} / c$. This requirement is not applied in the $\mu \tau_{\mu}$ channel as it favours the selection of $Z \rightarrow \mu^{+} \mu^{-}$background. The muon from $H \rightarrow \mu^{ \pm} \tau^{\mp}$ decay is expected to have a relatively large $p_{\mathrm{T}}$, thus the selection requires 
the muon $p_{\mathrm{T}}$ to be greater than $20 \mathrm{GeV} / c, 30 \mathrm{GeV} / c$, and $40 \mathrm{GeV} / c$ in the L-, $\mathrm{C}$-, and $\mathrm{H}$-selections, respectively. A tighter requirement of $50 \mathrm{GeV} / c$ is applied for the muon in the $\mu \tau_{\mu}$ channel in the H-selection due to the $Z \rightarrow \mu^{+} \mu^{-}$ background. Additionally, for the $\mu \tau_{e}$ channel, the contribution from $W / Z \rightarrow e+$ jet background is suppressed by requiring the transverse momentum of the muon to be larger than that of the $\tau_{e}$ candidate.

The relatively large lifetime of the $\tau$ lepton is used to suppress prompt background. For the $\tau_{h 3}$ candidate, a SV is reconstructed. A correction to the visible invariant mass, $m$, computed from the three-track combination, is obtained by exploiting the direction of flight defined from the PV to the $\mathrm{SV}$. The relation used is $m_{\text {corr }}=\sqrt{m^{2}+p^{2} \sin ^{2} \theta}+p \sin \theta$, where $\theta$ is the angle between the momentum of the $\tau_{h 3}$ candidate, and its flight direction. The $m_{\text {corr }}$ value is required to not exceed $3 \mathrm{GeV} / c^{2}$. A time-of-flight variable is also computed from the distance of flight and the partially reconstructed momentum of the $\tau$ lepton, and a minimum value of $30 \mathrm{fs}$ is required. The $m_{\text {corr }}$ and time-of-flight requirements together retain $80 \%$ of the signal, while rejecting about $75 \%$ of the QCD background. For tau decay channels with a single charged particle, it is not possible to reconstruct a SV, and a selection on the particle IP is applied. A threshold of IP $>10 \mu \mathrm{m}$ selects $85 \%$ of the $\tau_{e}$ and $\tau_{h 1}$ candidates, and rejects about $50 \%$ of the $V j$ background. The threshold is increased to $50 \mu \mathrm{m}$ for $\tau_{\mu}$ candidates, in order to suppress $Z \rightarrow \mu^{+} \mu^{-}$ background. The prompt muon instead is selected by requiring IP less than $50 \mu \mathrm{m}$, allowing up to $50 \%$ rejection of QCD and $Z \rightarrow \tau^{+} \tau^{-}$backgrounds.

The two leptons from the Higgs decay should be approximately back-to-back in the plane transverse to the beam. The absolute difference in azimuthal angle of muon and tau candidates is required to be greater than 2.7 radians. This rejects $50 \%$ of the $V j$ background. The transverse momentum asymmetry of the two particles, defined as $A_{p_{\mathrm{T}}}=$ $\left|p_{\mathrm{T} 1}-p_{\mathrm{T} 2}\right| /\left(p_{\mathrm{T} 1}+p_{\mathrm{T} 2}\right)$, can be used to effectively suppress various background processes. The background from the $V j$ processes is suppressed by up to $60 \%$ for the $\mu \tau_{h 1}$ channel by requiring $A_{p_{\mathrm{T}}}<0.4(0.5)$ in the L-selection (Sselection), because of the large $p_{\mathrm{T}}$ imbalance between the high- $p_{\mathrm{T}}$ muon from the vector boson and a hadron from a jet. For the $\mu \tau_{e}$ channel, the worse momentum resolution increases the average $A_{p_{\mathrm{T}}}$ value, hence a softer selection $A_{p_{\mathrm{T}}}<0.6$ is used to preserve efficiency. On the contrary, for the $\mu \tau_{\mu}$ channel, a tighter cut is applied to suppress the dominant background from $Z \rightarrow \mu^{+} \mu^{-}$decays. By requiring $A_{p_{\mathrm{T}}}>0.3(0.4)$ in the L-selection and C-selection $(\mathrm{H}-$ selection), such background is reduced by $80 \%$, while the signal decreases to $70 \%$.

The two leptons from the Higgs decay are required to be isolated from other charged particles. Two particleisolation variables are defined as $I_{p_{\mathrm{T}}}=\left(\vec{p}_{\text {cone }}\right)_{\mathrm{T}}$ and $\hat{I}_{p_{\mathrm{T}}}=$
$p_{\mathrm{T}} /\left(\vec{p}+\vec{p}_{\text {cone }}\right)_{\mathrm{T}}$ where $\vec{p}$ is the momentum of the lepton candidate, the subscript $\mathrm{T}$ denotes the component in the transverse plane, and $\vec{p}_{\text {cone }}$ is the sum of the momenta of all charged tracks within a distance $R_{\eta \phi}=0.5$ in the $(\eta, \phi)$ plane around the lepton candidate. The isolation requirement $\hat{I}_{p_{\mathrm{T}}}>0.9$ is applied to the muon and tau candidates for all decay channels and selection sets, and retain $70 \%$ of the signal candidates while rejecting $90 \%$ of QCD events. In addition, a cut $I_{p_{\mathrm{T}}}<2 \mathrm{GeV} / c$ is applied in the L-selection to both candidates, as the lower $p_{\mathrm{T}}$ reduces the background rejection power of the $\hat{I}_{p_{\mathrm{T}}}$ variable.

The selection criteria common or specific to each selection set and decay channel are summarised in Table 1. The signal selection efficiencies are found to vary from 10 to $50 \%$. Due to the kinematic selection, the decay channels are mutually exclusive and just one $\mu^{ \pm} \tau^{\mp}$ candidate per event is found.

\section{Background estimation}

Several background processes are considered: $Z \rightarrow \tau^{+} \tau^{-}$, $Z \rightarrow l^{+} l^{-}(l=e, \mu), \mathrm{QCD}, V j$, double bosons production $(V V), t \bar{t}$, and $Z \rightarrow b \bar{b}$. All backgrounds except $Z \rightarrow \tau^{+} \tau^{-}$ are estimated following the procedures described in Ref. [37]. The expected yields can be found in Table 2 . The corresponding invariant-mass distributions compared with candidates observed in the data are shown in Fig. 1. For illustration, examples of $H \rightarrow \mu^{ \pm} \tau^{\mp}$ distributions from simulation are also superimposed.

The $Z \rightarrow \tau^{+} \tau^{-}$background is estimated from the crosssection measured by the LHCb collaboration [37] where the reconstruction efficiency is determined from data, and the acceptance and selection efficiency are obtained from simulation. The estimated background includes a small amount of cross-feed from different final states of the tau decay, as determined from simulation. The $Z \rightarrow \mu^{+} \mu^{-}$background is dominant in the $\mu \tau_{\mu}$ channel. The corresponding invariantmass distribution is obtained from simulation and normalised to data in the $Z$ peak region, from 80 to $100 \mathrm{GeV} / c^{2}$. In order to suppress the potential presence of signal in this region, the muons are required to be promptly produced. For other channels, the $Z \rightarrow l^{+} l^{-}$decay becomes a background source in case a lepton is misidentified. This contribution is computed from the $Z \rightarrow l^{+} l^{-}$in data, and weighted by the particle misidentification probability obtained from simulation.

The QCD and $V j$ backgrounds are inferred from data using the same criteria as for the signal but selecting same$\operatorname{sign} \mu^{ \pm} \tau^{ \pm}$candidates. Their amounts are determined by a fit to the distribution of $p_{\mathrm{T}}(\mu)-p_{\mathrm{T}}(\tau)$, with templates representing each of them. The template for the QCD component is obtained from data requiring an anti-isolation $\hat{I}_{p_{\mathrm{T}}}<0.6$ selection. The distribution obtained from simulation is used for the $V j$ component. Factors are subsequently applied for 
Table 1 Requirements for each decay channel and selection set

\begin{tabular}{|c|c|c|c|c|c|}
\hline Selection set & Variable & $\mu \tau_{e}$ & $\mu \tau_{h 1}$ & $\mu \tau_{h 3}$ & $\mu \tau_{\mu}$ \\
\hline \multirow[t]{13}{*}{ All } & $p_{\mathrm{T}}(\tau)[\mathrm{GeV} / c]$ & $>5$ & $>10$ & $>12$ & $>5$ \\
\hline & $p_{\mathrm{T}}\left(\tau_{h 3}^{\text {prong1 }}\right)[\mathrm{GeV} / c]$ & - & - & $>1$ & - \\
\hline & $p_{\mathrm{T}}\left(\tau_{h 3}^{\text {prong2 }}\right)[\mathrm{GeV} / c]$ & - & - & $>1$ & - \\
\hline & $p_{\mathrm{T}}\left(\tau_{h 3}^{\text {prong3 }}\right)[\mathrm{GeV} / c]$ & - & - & $>6$ & - \\
\hline & $p_{\mathrm{T}}(\mu)-p_{\mathrm{T}}(\tau)[\mathrm{GeV} / c]$ & $>0$ & - & - & - \\
\hline & $m\left(\tau_{h 3}\right)\left[\mathrm{GeV} / c^{2}\right]$ & - & - & $0.7-1.5$ & - \\
\hline & $m_{\mathrm{corr}}\left(\tau_{h 3}\right)\left[\mathrm{GeV} / c^{2}\right]$ & - & - & $>3$ & - \\
\hline & Time-of-flight $\left(\tau_{h 3}\right)[\mathrm{fs}]$ & - & - & $>30$ & - \\
\hline & $\operatorname{IP}(\tau)[\mu \mathrm{m}]$ & $>10$ & $>10$ & - & $>50$ \\
\hline & $\mathrm{IP}(\mu)[\mu \mathrm{m}]$ & $<50$ & $<50$ & $<50$ & $<50$ \\
\hline & $\Delta \phi[\mathrm{rad}]$ & $>2.7$ & $>2.7$ & $>2.7$ & $>2.7$ \\
\hline & $\hat{I}_{p_{\mathrm{T}}}(\tau)$ & $>0.9$ & $>0.9$ & $>0.9$ & $>0.9$ \\
\hline & $\hat{I}_{p_{\mathrm{T}}}(\mu)$ & $>0.9$ & $>0.9$ & $>0.9$ & $>0.9$ \\
\hline \multirow[t]{4}{*}{ L-selection } & $p_{\mathrm{T}}(\mu)[\mathrm{GeV} / c]$ & $>20$ & $>20$ & $>20$ & $>20$ \\
\hline & $A_{p_{\mathrm{T}}}$ & $<0.6$ & $<0.4$ & - & $>0.3$ \\
\hline & $I_{p_{\mathrm{T}}}(\tau)[\mathrm{GeV} / c]$ & $<2$ & $<2$ & $<2$ & $<2$ \\
\hline & $I_{p_{\mathrm{T}}}(\mu)[\mathrm{GeV} / c]$ & $<2$ & $<2$ & $<2$ & $<2$ \\
\hline \multirow[t]{2}{*}{ C-selection } & $p_{\mathrm{T}}(\mu)[\mathrm{GeV} / c]$ & $>30$ & $>30$ & $>30$ & $>30$ \\
\hline & $A_{p_{\mathrm{T}}}$ & - & $<0.5$ & - & $>0.3$ \\
\hline \multirow[t]{3}{*}{ H-selection } & $p_{\mathrm{T}}(\tau)[\mathrm{GeV} / c]$ & $>20$ & $>20$ & $>20$ & - \\
\hline & $p_{\mathrm{T}}(\mu)[\mathrm{GeV} / c]$ & $>40$ & $>40$ & $>40$ & $>50$ \\
\hline & $A_{p_{\mathrm{T}}}$ & - & - & - & $>0.4$ \\
\hline
\end{tabular}

the correction of the relative yield of opposite-sign to samesign candidates. For the QCD background the number of anti-isolated opposite-sign candidates found in data is used in the calculation of the correction factor, where it is found to be close to unity. The factors are found consistent with the simulation. The factors for the $V j$ component are taken from simulation, and are in general larger than unity (1.3 for $\mu \tau_{e}$ up to 3.1 for $\mu \tau_{h 1}$, for the L-selection). The minor contributions from $V V, t \bar{t}$, and $Z \rightarrow b \bar{b}$ processes are estimated from simulation.

\section{Results}

The signal cross-section multiplied by the branching fraction is given by

$\sigma\left(g g \rightarrow H \rightarrow \mu^{ \pm} \tau^{\mp}\right)=N_{\text {sig }} /(\mathcal{L} \cdot \mathcal{B}(\tau \rightarrow X) \cdot \varepsilon)$,

where $N_{\text {sig }}$ is the signal yield obtained from the fit procedure described below, $\mathcal{L}$ the total integrated luminosity, $\mathcal{B}(\tau \rightarrow X)$ the tau branching fraction, and $\varepsilon$ the detection efficiency. The latter is the product of acceptance, reconstruction, and offline selection efficiencies. These efficiencies are obtained from simulated samples and data for each decay channel and selection set, following the methods developed for the $Z \rightarrow \tau^{+} \tau^{-}$measurement [37]. The acceptance obtained from the POWHEG- BOX generator is identical for the $\mu \tau_{e}, \mu \tau_{h 3}$, and $\mu \tau_{\mu}$ channels, varying from $1.0 \%$ for $m_{H}=195 \mathrm{GeV} / c^{2}$ to $3.2 \%$ for $m_{H}=75 \mathrm{GeV} / c^{2}$. The reconstruction efficiency, which is the product of contributions from trigger, tracking, and particle identification, is in the range $40-70 \%$, but only about $15 \%$ in the case of the $\mu \tau_{h 3}$ channel because of the limited tracking efficiency for the lowmomentum hadrons. With the exception of the $\mu \tau_{\mu}$ channel, the selection efficiency is $18-30 \%$ in the L-selection, and $24-49 \%$ in the C-selection and H-selection. In the case of the $\mu \tau_{\mu}$ channel, the tighter selection on the muon $p_{\mathrm{T}}$ and impact parameter reduces the selection efficiency to $10-15 \%$.

The systematic uncertainties are summarised in Table 3. The uncertainty on the acceptance receives contributions from the gluon PDF uncertainty, as well as from factorization and renormalisation scales. The uncertainties on the reconstruction and selection efficiencies are estimated from simulation and are calibrated using data as described in Ref. [37]. The uncertainty associated with the invariant-mass shape is handled by selecting the weakest expected limits among the different choices of distribution (kernel estimation and histograms with different bin widths are used). The uncertainties on the integrated luminosity and acceptance are fully 
Table 2 Expected number of background candidates from each component, total background with uncertainty, and number of observed candidates with statistical uncertainty, from each decay channel and selection set

\begin{tabular}{|c|c|c|c|c|c|}
\hline Selection set & Process & $\mu \tau_{e}$ & $\mu \tau_{h 1}$ & $\mu \tau_{h 3}$ & $\mu \tau_{\mu}$ \\
\hline \multirow[t]{9}{*}{ L-selection } & $Z \rightarrow \tau^{+} \tau^{-}$ & $371.1 \pm 26.0$ & $681.7 \pm 47.1$ & $135.1 \pm 11.7$ & $137.4 \pm 9.5$ \\
\hline & $Z \rightarrow l^{+} l^{-}$ & $8.2 \pm 1.6$ & $4.0 \pm 1.8$ & - & $155.3 \pm 5.0$ \\
\hline & QCD & $67.5 \pm 10.6$ & $463.6 \pm 5.4$ & $93.1 \pm 10.9$ & $19.4 \pm 5.5$ \\
\hline & $V j$ & $14.5 \pm 10.3$ & $143.2 \pm 58.6$ & $40.1 \pm 15.8$ & $10.7 \pm 5.8$ \\
\hline & VV & $3.4 \pm 0.3$ & $0.9 \pm 0.2$ & $0.3 \pm 0.1$ & $0.3 \pm 0.1$ \\
\hline & $t \bar{t}$ & $1.7 \pm 0.1$ & $1.3 \pm 0.1$ & $0.7 \pm 0.1$ & $1.3 \pm 0.2$ \\
\hline & $Z \rightarrow b \bar{b}$ & $0.2 \pm 0.2$ & $0.2 \pm 0.2$ & $0.1 \pm 0.1$ & $0.2 \pm 0.2$ \\
\hline & Total background & $466.6 \pm 28.0$ & $1294.9 \pm 75.5$ & $269.4 \pm 20.3$ & $324.5 \pm 12.5$ \\
\hline & Observed & $472.0 \pm 21.7$ & $1284.0 \pm 35.8$ & $240.0 \pm 15.5$ & $344.0 \pm 18.5$ \\
\hline \multirow[t]{9}{*}{ C-selection } & $Z \rightarrow \tau^{+} \tau^{-}$ & $200.0 \pm 14.3$ & $288.1 \pm 20.2$ & $61.3 \pm 5.5$ & $71.7 \pm 5.2$ \\
\hline & $Z \rightarrow l^{+} l^{-}$ & $8.0 \pm 1.7$ & $4.3 \pm 1.8$ & - & $126.7 \pm 4.5$ \\
\hline & QCD & $10.0 \pm 14.0$ & $137.9 \pm 14.0$ & $29.9 \pm 9.0$ & $6.1 \pm 3.6$ \\
\hline & $V j$ & $48.3 \pm 17.2$ & $242.9 \pm 25.3$ & $30.8 \pm 17.6$ & $7.9 \pm 4.7$ \\
\hline & VV & $3.4 \pm 0.3$ & $1.5 \pm 0.2$ & $0.3 \pm 0.1$ & $0.3 \pm 0.1$ \\
\hline & $t \bar{t}$ & $2.5 \pm 0.1$ & $1.6 \pm 0.1$ & $0.7 \pm 0.1$ & $1.5 \pm 0.2$ \\
\hline & $Z \rightarrow b \bar{b}$ & $0.1 \pm 0.1$ & $0.1 \pm 0.1$ & $0.1 \pm 0.1$ & $0.1 \pm 0.1$ \\
\hline & Total background & $272.3 \pm 17.8$ & $676.4 \pm 35.2$ & $123.1 \pm 15.0$ & $214.3 \pm 8.1$ \\
\hline & Observed & $296.0 \pm 17.2$ & $679.0 \pm 26.1$ & $123.0 \pm 11.1$ & $235.0 \pm 15.3$ \\
\hline \multirow[t]{9}{*}{ H-selection } & $Z \rightarrow \tau^{+} \tau^{-}$ & $13.7 \pm 1.8$ & $18.4 \pm 1.6$ & $8.9 \pm 1.1$ & $2.2 \pm 0.4$ \\
\hline & $Z \rightarrow l^{+} l^{-}$ & $4.7 \pm 1.1$ & $2.5 \pm 1.1$ & - & $33.7 \pm 2.3$ \\
\hline & QCD & - & $15.8 \pm 6.3$ & $9.7 \pm 5.1$ & - \\
\hline & $V j$ & $3.5 \pm 2.6$ & $142.6 \pm 26.0$ & $18.6 \pm 16.5$ & $7.8 \pm 4.0$ \\
\hline & VV & $1.7 \pm 0.2$ & $1.0 \pm 0.2$ & $0.1 \pm 0.1$ & $0.2 \pm 0.1$ \\
\hline & $t \bar{t}$ & $1.2 \pm 0.1$ & $0.9 \pm 0.1$ & $0.4 \pm 0.1$ & $0.8 \pm 0.1$ \\
\hline & $Z \rightarrow b \bar{b}$ & $0.1 \pm 0.1$ & $0.1 \pm 0.1$ & $0.1 \pm 0.1$ & $0.1 \pm 0.1$ \\
\hline & Total background & $24.9 \pm 3.4$ & $181.2 \pm 26.7$ & $37.8 \pm 13.6$ & $44.7 \pm 4.6$ \\
\hline & Observed & $27.0 \pm 5.2$ & $184.0 \pm 13.6$ & $37.0 \pm 6.1$ & $39.0 \pm 6.2$ \\
\hline
\end{tabular}

correlated among channels, while only a partial correlation is found for the reconstruction efficiency uncertainties. All the other uncertainties are taken as uncorrelated.

The signal yield is determined from a simultaneous extended likelihood fit of the binned invariant-mass distributions of the $\mu \tau$ candidates. The distributions for signal are obtained from simulation, while distributions of the different background sources are obtained using the method described in Sect. 4. The amount of each background component as well as other terms in Eq. (1) containing uncertainties are treated as nuisance parameters and are constrained to a Gaussian distribution with mean and standard deviation corresponding to the expected value and its uncertainty, respectively.

The fit results for all $m_{H}$ values are compatible with a null signal, hence cross-section upper limits are computed. The exclusion limits of $\sigma\left(g g \rightarrow H \rightarrow \mu^{ \pm} \tau^{\mp}\right)$ defined at $95 \%$ confidence level are obtained from the $\mathrm{CL}_{\mathrm{s}}$ method [56]. As mentioned before, for each mass hypothesis the selection considered is that providing the smallest expected limit. The $\sigma\left(g g \rightarrow H \rightarrow \mu^{ \pm} \tau^{\mp}\right)$ exclusion limits are shown in Fig. 2, ranging from $22 \mathrm{pb}$ for $m_{H}=45 \mathrm{GeV} / c^{2}$ to $4 \mathrm{pb}$ for $m_{H}$ $=195 \mathrm{GeV} / c^{2}$. In the particular case of $m_{H}=125 \mathrm{GeV} / c^{2}$, using the production cross-section from Ref. [57] gives a best fit for the branching fraction of $\mathcal{B}\left(H \rightarrow \mu^{ \pm} \tau^{\mp}\right)=-2_{-12}^{+14} \%$ and an observed exclusion limit $\mathcal{B}\left(H \rightarrow \mu^{ \pm} \tau^{\mp}\right)<26 \%$. The corresponding exclusion limit on the Yukawa coupling is $\sqrt{\left|Y_{\mu \tau}\right|^{2}+\left|Y_{\tau \mu}\right|^{2}}<1.7 \times 10^{-2}$, assuming the decay width $\Gamma_{\mathrm{SM}}=4.1 \mathrm{MeV} / c^{2}[58]$.

\section{Conclusion}

A search for Higgs-like bosons decaying via a lepton-flavourviolating process $H \rightarrow \mu^{ \pm} \tau^{\mp}$ in $p p$ collisions at $\sqrt{s}=8 \mathrm{TeV}$ is presented, with the tau lepton reconstructed in leptonic and hadronic decay modes. No signal has been found. The upper bound on the cross-section multiplied by the branching fraction, at $95 \%$ confidence level, ranges from $22 \mathrm{pb}$ for a boson 

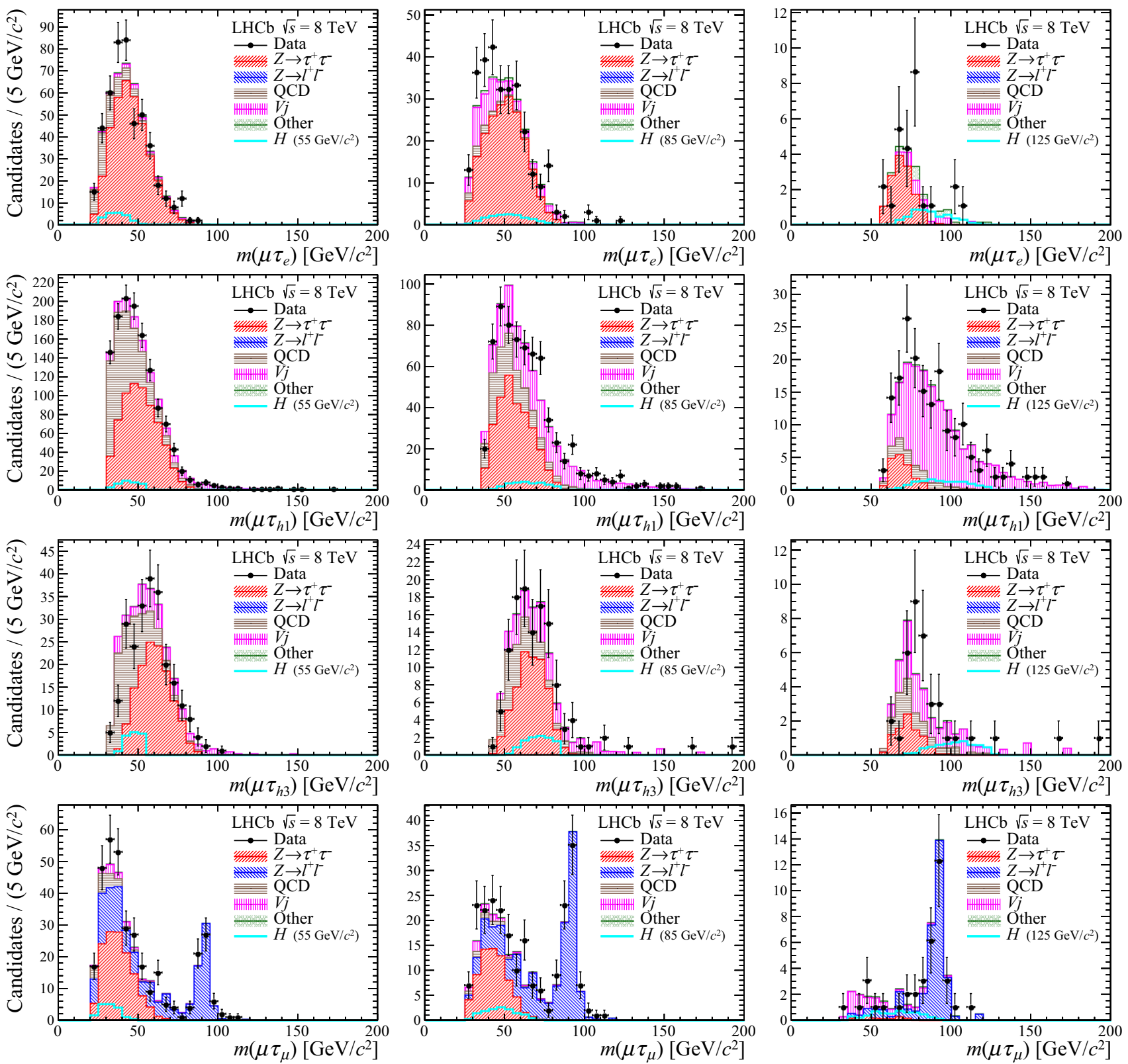

Fig. 1 Invariant-mass distributions for the $\mu^{ \pm} \tau^{\mp}$ candidates for the four decay channels (from top to bottom: $\mu \tau_{e}, \mu \tau_{h 1}, \mu \tau_{h 3}, \mu \tau_{\mu}$ ) and the three selections (from left to right: L-selection, C-selection, $\mathrm{H}$ selection). The distribution of candidates observed (black points) is compared with backgrounds (filled colour, stacked), and with signal hypothesis (cyan). The signal is normalised to $\sqrt{N}$, with $N$ the total number of candidates in the corresponding data histogram
Table 3 Relative systematic uncertainties (in \%) on the normalisation factors in the cross-section calculation. When the uncertainty depends on $m_{H}$ a range is indicated

\begin{tabular}{lllll}
\hline & $\mu \tau_{e}$ & $\mu \tau_{h 1}$ & $\mu \tau_{h 3}$ & $\mu \tau_{\mu}$ \\
\hline Luminosity & 1.16 & 1.16 & 1.16 & 1.16 \\
Tau branching fraction & 0.22 & 0.18 & 0.48 & 0.23 \\
PDF & $2.6-7.1$ & $3.5-7.2$ & $2.6-7.3$ & $3.0-7.9$ \\
Scales & $0.9-1.9$ & $0.8-1.7$ & $0.9-1.7$ & $0.9-1.9$ \\
Reconstruction efficiency & $1.8-3.6$ & $1.9-5.4$ & $3.3-7.1$ & $1.5-3.3$ \\
Selection efficiency & $2.5-6.0$ & $1.9-4.1$ & $4.0-9.3$ & $3.8-8.5$ \\
\hline
\end{tabular}




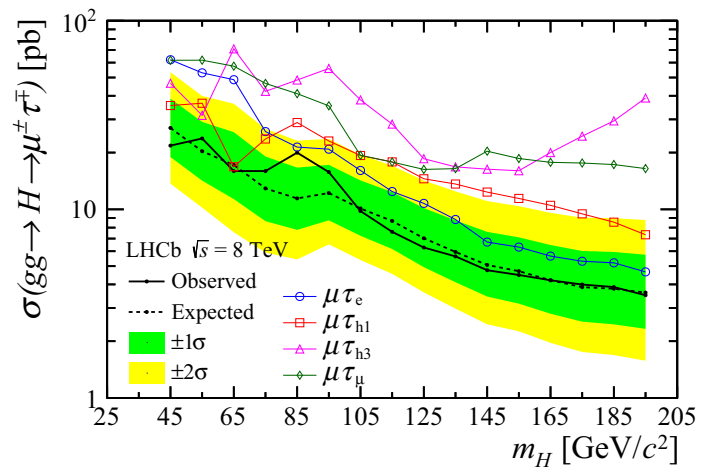

Fig. 2 Cross-section times branching fraction 95\% CL limits for the $H \rightarrow \mu^{ \pm} \tau^{\mp}$ decay as a function of $m_{H}$, from the simultaneous fit. The observed limits from individual channels are also shown

mass of $45 \mathrm{GeV} / c^{2}$, to $4 \mathrm{pb}$ for $195 \mathrm{GeV} / c^{2}$. The search provides information complementary to the ATLAS and CMS collaborations.

Acknowledgements We express our gratitude to our colleagues in the CERN accelerator departments for the excellent performance of the LHC. We thank the technical and administrative staff at the LHCb institutes. We acknowledge support from CERN and from the national agencies: CAPES, CNPq, FAPERJ and FINEP (Brazil); MOST and NSFC (China); CNRS/IN2P3 (France); BMBF, DFG and MPG (Germany); INFN (Italy); NWO (The Netherlands); MNiSW and NCN (Poland); MEN/IFA (Romania); MSHE (Russia); MinECo (Spain); SNSF and SER (Switzerland); NASU (Ukraine); STFC (United Kingdom); NSF (USA). We acknowledge the computing resources that are provided by CERN, IN2P3 (France), KIT and DESY (Germany), INFN (Italy), SURF (The Netherlands), PIC (Spain), GridPP (United Kingdom), RRCKI and Yandex LLC (Russia), CSCS (Switzerland), IFINHH (Romania), CBPF (Brazil), PL-GRID (Poland) and OSC (USA). We are indebted to the communities behind the multiple open-source software packages on which we depend. Individual groups or members have received support from AvH Foundation (Germany); EPLANET, Marie Skłodowska-Curie Actions and ERC (European Union); ANR, Labex P2IO and OCEVU, and Région Auvergne-Rhône-Alpes (France); Key Research Program of Frontier Sciences of CAS, CAS PIFI, and the Thousand Talents Program (China); RFBR, RSF and Yandex LLC (Russia); GVA, XuntaGal and GENCAT (Spain); the Royal Society and the Leverhulme Trust (United Kingdom); Laboratory Directed Research and Development program of LANL (USA).

Open Access This article is distributed under the terms of the Creative Commons Attribution 4.0 International License (http://creativecomm ons.org/licenses/by/4.0/), which permits unrestricted use, distribution, and reproduction in any medium, provided you give appropriate credit to the original author(s) and the source, provide a link to the Creative Commons license, and indicate if changes were made.

Funded by SCOAP ${ }^{3}$.

\section{References}

1. M. Blanke et al., $\Delta F=2$ observables and fine-tuning in a warped extra dimension with custodial protection. JHEP 03, 001 (2009). arXiv:0809.1073

2. G.F. Giudice, O. Lebedev, Higgs-dependent Yukawa couplings. Phys. Lett. B 665, 79 (2008). arXiv:0804.1753
3. J.A. Aguilar-Saavedra, A minimal set of top-Higgs anomalous couplings. Nucl. Phys. B 821, 215 (2009). arXiv:0904.2387

4. M.E. Albrecht et al., Electroweak and flavour structure of a warped extra dimension with custodial protection. JHEP 09, 064 (2009). arXiv:0903.2415

5. A. Goudelis, O. Lebedev, J-h Park, Higgs-induced lepton flavour violation. Phys. Lett. B 707, 369 (2012). arXiv:1111.1715

6. D. McKeen, M. Pospelov, A. Ritz, Modified Higgs branching ratios versus CP and lepton flavour violation. Phys. Rev. D 86, 113004 (2012). arXiv:1208.4597

7. E. Arganda, A.M. Curiel, M.J. Herrero, D. Temes, Lepton flavour violating Higgs boson decays from massive seesaw neutrinos. Phys. Rev. D 71, 035011 (2005). arXiv:hep-ph/0407302

8. E. Arganda, M.J. Herrero, X. Marcano, C. Weiland, Imprints of massive inverse seesaw model neutrinos in lepton flavour violating Higgs boson decays. Phys. Rev. D 91, 015001 (2015). arXiv: 1405.4300

9. R. Harnik, J. Kopp, J. Zupan, Flavour violating Higgs decays. JHEP 03, 026 (2013). arXiv: 1209.1397

10. J.D. Bjorken, S. Weinberg, A mechanism for nonconservation of muon number. Phys. Rev. Lett. 38, 622 (1977)

11. J.L. Diaz-Cruz, J.J. Toscano, Lepton flavour violating decays of Higgs bosons beyond the standard model. Phys. Rev. D 62, 116005 (2000). arXiv:hep-ph/9910233

12. T. Han, D. Marfatia, $H \rightarrow \mu \tau$ at hadron colliders. Phys. Rev. Lett. 86, 1442 (2001). arXiv:hep-ph/0008141

13. A. Arhrib, Y. Cheng, O.C.W. Kong, Comprehensive analysis on lepton flavour violating Higgs boson to $\mu^{\mp} \tau^{ \pm}$decay in supersymmetry without $R$ parity. Phys. Rev. D 87, 015025 (2013). arXiv:1210.8241

14. M. Arana-Catania, E. Arganda, M.J. Herrero, Non-decoupling SUSY in LFV Higgs decays: a window to new physics at the LHC. JHEP 09, 160 (2013) [Erratum ibid 10, 192 (2015)]. arXiv: 1304.3371

15. K. Agashe, R. Contino, Composite Higgs-mediated flavorchanging neutral current. Phys. Rev. D 80, 075016 (2009). arXiv:0906.1542

16. A. Azatov, M. Toharia, L. Zhu, Higgs mediated flavor-changing neutral current's in warped extra dimensions. Phys. Rev. D 80, 035016 (2009). arXiv:0906.1990

17. G. Perez, L. Randall, Natural neutrino masses and mixings from warped geometry. JHEP 01, 077 (2009). arXiv:0805.4652

18. S. Casagrande, Flavour physics in the Randall-Sundrum model: I. Theoretical setup and electroweak precision tests, JHEP 10, 094 (2008). arXiv:0807.4937

19. H. Ishimori et al., Non-Abelian discrete symmetries in particle physics. Prog. Theor. Phys. Suppl. 183, 1 (2010). arXiv:1003.3552

20. ALEPH collaboration, D. Decamp et al., Searches for new particles in $Z$ decays using the ALEPH detector. Phys. Rep. 216, 253 (1992)

21. DELPHI collaboration, P. Abreu et al., A search for lepton flavour violation in $Z$ decays. Phys. Lett. B 298, 247 (1993)

22. L3 collaboration, O. Adriani et al., Search for lepton flavour violation in $Z$ decays. Phys. Lett. B 316, 427 (1993)

23. OPAL collaboration, M.Z. Akrawy et al., A Search for lepton flavour violation in $Z$ decays. Phys. Lett. B 254, 293 (1991)

24. OPAL collaboration, G. Abbiendi et al., Search for lepton flavour violation in $e^{+} e^{-}$collisions at $\sqrt{s}=189-209 \mathrm{GeV}$. Phys. Lett. B 519, 23 (2001). arXiv:hep-ex/0109011

25. G. Blankenburg, J. Ellis, G. Isidori, Flavour-changing decays of a $125 \mathrm{GeV}$ Higgs-like particle. Phys. Lett. B 712, 386 (2012). arXiv: 1202.5704

26. CMS collaboration, A.M. Sirunyan et al., Search for lepton flavour violating decays of the Higgs boson to $\mu \tau$ and e $\tau$ in proton-proton collisions at $\sqrt{s}=13$ TeV. JHEP 06, 001 (2018). arXiv: 1712.07173

27. ATLAS collaboration, G. Aad et al., Search for lepton-flavourviolating $H \rightarrow \mu \tau$ decays of the Higgs boson with the ATLAS detector. JHEP 11, 211 (2015). arXiv: 1508.03372 
28. J.F. Gunion, H.E. Haber, The CP-conserving two-Higgs doublet model: the approach to the decoupling limit. Phys. Rev. D 67, 075019 (2003). arXiv:hep-ph/0207010

29. ATLAS collaboration, G. Aad et al., Search for the neutral Higgs bosons of the Minimal Supersymmetric Standard Model in $p p$ collisions at $\sqrt{s}=7 \mathrm{TeV}$ with the ATLAS detector. JHEP 02, 095 (2013). arXiv: 1211.6956

30. CMS collaboration, S. Chatrchyan et al., Search for neutral Higgs bosons decaying to tau pairs in $p p$ collisions at $\sqrt{s}=7 \mathrm{TeV}$. Phys. Lett. B 713, 68 (2012). arXiv: 1202.4083

31. J. Jaeckel, A. Ringwald, The low-energy frontier of particle physics. Annu. Rev. Nucl. Part. Sci. 60, 405 (2010). arXiv: 1002.0329

32. M. Baumgart et al., Non-Abelian dark sectors and their collider signatures. JHEP 04, 014 (2009). arXiv:0901.028

33. BaBar collaboration, J.P. Lees et al., Search for low-mass darksector Higgs bosons. Phys. Rev. Lett. 108, 211801 (2012). arXiv: 1202.1313

34. Belle Collaboration, I. Jaegle, Search for the 'Dark Photon' and the 'Dark Higgs' at Belle. Nucl. Phys. Proc. Suppl. 234, 33 (2013). arXiv: 1211.1403

35. LHCb Collaboration, R. Aaij et al., Search for dark photons produced in $13 \mathrm{TeV} p p$ collisions. Phys. Rev. Lett. 120, 061801 (2018). arXiv: 1710.02867

36. H.M. Georgi, S.L. Glashow, M.E. Machacek, D.V. Nanopoulos, Higgs bosons from two-gluon annihilation in proton-proton collisions. Phys. Rev. Lett. 40, 692 (1978)

37. LHCb Collaboration, R. Aaij et al., Measurement of $Z \rightarrow \tau^{+} \tau^{-}$ production in proton-proton collisions at $\sqrt{s}=8 \mathrm{TeV}$. JHEP 09, 159 (2018). arXiv:1806.05008

38. LHCb Collaboration, A.A. Alves Jr. et al., The LHCb detector at the LHC. JINST 3, S08005 (2008)

39. LHCb Collaboration, R. Aaij et al., LHCb detector performance. Int. J. Mod. Phys. A 30, 1530022 (2015). arXiv:1412.6352

40. T. Sjöstrand, S. Mrenna, P. Skands, A brief introduction to PYTHIA 8.1. Comput. Phys. Commun. 178, 852 (2008). arXiv:0710.3820

41. T. Sjöstrand, S. Mrenna, P. Skands, PYTHIA 6.4 physics and manual. JHEP 05, 026 (2006). arXiv:hep-ph/0603175

42. I. Belyaev et al., Handling of the generation of primary events in Gauss, the LHCb simulation framework. J. Phys. Conf. Ser. 331, 032047 (2011)
43. J. Pumplin et al., New generation of parton distributions with uncertainties from global QCD analysis. JHEP 07, 012 (2002). arXiv:hep-ph/0201195

44. D.J. Lange, The EvtGen particle decay simulation package. Nucl. Instrum. Methods A 462, 152 (2001)

45. P. Golonka, Z. Was, PHOTOS Monte Carlo: a precision tool for QED corrections in $Z$ and $W$ decays. Eur. Phys. J. C 45, 97 (2006). arXiv:hep-ph/0506026

46. Geant4 Collaboration, J. Allison et al., Geant4 developments and applications. IEEE Trans. Nucl. Sci. 53, 270 (2006)

47. Geant4 Collaboration, S. Agostinelli et al., Geant4: a simulation toolkit. Nucl. Instrum. Methods A 506, 250 (2003)

48. M. Clemencic, The LHCb simulation application, Gauss: design, evolution and experience. J. Phys. Conf. Ser. 331, 032023 (2011)

49. P. Nason, A new method for combining NLO QCD with shower Monte Carlo algorithms. JHEP 11, 040 (2004). arXiv:hep-ph/0409146

50. S. Frixione, P. Nason, C. Oleari, Matching NLO QCD computations with parton shower simulations: the POWHEG method. JHEP 11, 070 (2007). arXiv:0709.2092

51. S. Alioli, P. Nason, C. Oleari, E. Re, A general framework for implementing NLO calculations in shower Monte Carlo programs: the POWHEG BOX. JHEP 06, 043 (2010). arXiv:1002.2581

52. S. Alioli, P. Nason, C. Oleari, E. Re, NLO Higgs boson production via gluon fusion matched with shower in POWHEG. JHEP 04, 002 (2009). arXiv:0812.0578

53. L.A. Harland-Lang, A.D. Martin, P. Motylinski, R.S. Thorne, Parton distributions in the LHC era: MMHT 2014 PDFs. Eur. Phys. J. C 75, 204 (2015). arXiv: 1412.3989

54. LHCb collaboration, R. Aaij et al., Precision luminosity measurements at LHCb. JINST 9, P12005 (2014). arXiv:1410.0149

55. F. Machefert, LHCb calorimeters and muon system lepton identification, in AIP Conference Proceedings. AIP (2004). https://doi. org/10.1063/1.1807309

56. A.L. Read, Presentation of search results: the $C L_{s}$ technique. J. Phys. G 28, 2693 (2002)

57. LHC Higgs Cross Section Working Group, J.R. Andersen et al., Handbook of LHC Higgs cross sections: 3. Higgs properties. Tech. rep., CERN, CERN-2013-004 (2013)

58. A. Denner, Standard model Higgs-boson branching ratios with uncertainties. Eur. Phys. J. C 71, 1753 (2011). arXiv:1107.5909

\section{LHCb Collaboration ${ }^{\star}$}

R. Aaij ${ }^{27}$, C. Abellán Beteta ${ }^{44}$, B. Adeva ${ }^{41}$, M. Adinolfi ${ }^{48}$, C. A. Aidala ${ }^{73}$, Z. Ajaltouni ${ }^{5}$, S. Akar ${ }^{59}$, P. Albicocco ${ }^{18}$, J. Albrecht ${ }^{10}, \mathrm{~F}$. Alessio ${ }^{42}$, M. Alexander ${ }^{53}$, A. Alfonso Albero ${ }^{40}$, G. Alkhazov ${ }^{33}$, P. Alvarez Cartelle ${ }^{55}$, A. A. Alves Jr ${ }^{41}$, S. Amato ${ }^{2}$, S. Amerio ${ }^{23}$, Y. Amhis ${ }^{7}$, L. An ${ }^{3}$, L. Anderlini ${ }^{17}$, G. Andreassi4 ${ }^{43}$, M. Andreotti 16,g, J. E. Andrews ${ }^{60}$, R. B. Appleby ${ }^{56}$, F. Archilli ${ }^{27}$, P. d'Argent ${ }^{12}$, J. Arnau Romeu ${ }^{6}$, A. Artamonov ${ }^{39}$, M. Artuso ${ }^{61}$, K. Arzymatov ${ }^{37}$, E. Aslanides ${ }^{6}$, M. Atzeni ${ }^{44}$, B. Audurier ${ }^{22}$ ，S. Bachmann ${ }^{12}$, J. J. Back ${ }^{50}$ S. Baker ${ }^{55}$, V. Balagura ${ }^{7, b}$, W. Baldini ${ }^{16}$, A. Baranov ${ }^{37}$, R. J. Barlow ${ }^{56}$, S. Barsuk ${ }^{7}$, W. Barter ${ }^{56}$, F. Baryshnikov ${ }^{70}$, V. Batozskaya ${ }^{31}$, B. Batsukh ${ }^{61}$, V. Battista ${ }^{43}$, A. Bay ${ }^{43}$, J. Beddow ${ }^{53}$, F. Bedeschi ${ }^{24}$, I. Bediaga ${ }^{1}$, A. Beiter ${ }^{61}$, L. J. Bel ${ }^{27}$, S. Belin ${ }^{22}$, N. Beliy ${ }^{63}$, V. Bellee ${ }^{43}$, N. Belloli ${ }^{20, i}$, K. Belous ${ }^{39}$, I. Belyaev ${ }^{34,42}$, E. Ben-Haim ${ }^{8}$, G. Bencivenni ${ }^{18}$, S. Benson ${ }^{27}$, S. Beranek ${ }^{9}$, A. Berezhnoy ${ }^{35}$, R. Bernet ${ }^{44}$, D. Berninghoff ${ }^{12}$, E. Bertholet ${ }^{8}$, A. Bertolin ${ }^{23}$, C. Betancourt ${ }^{44}$, F. Betti ${ }^{15,42}$, M.O. Bettler ${ }^{49}$, M. van Beuzekom ${ }^{27}$, Ia. Bezshyiko ${ }^{44}$, S. Bhasin ${ }^{48}$, J. Bhom ${ }^{29}$, S. Bifani ${ }^{47}$, P. Billoir ${ }^{8}$, A. Birnkraut ${ }^{10}$, A. Bizzeti ${ }^{17, u}$, M. Bjørn ${ }^{57}$, M. P. Blago ${ }^{42}$, T. Blake ${ }^{50}$, F. Blanc ${ }^{43}$, S. Blusk ${ }^{61}$, D. Bobulska ${ }^{53}$, V. Bocci ${ }^{26}$, O. Boente Garcia ${ }^{41}$, T. Boettcher ${ }^{58}$, A. Bondar ${ }^{38, w}$, N. Bondar ${ }^{33}$, S. Borghi ${ }^{42,56}$, M. Borisyak ${ }^{37}$, M. Borsato ${ }^{41}$, F. Bossu ${ }^{7}$, M. Boubdir ${ }^{9}$, T. J. V. Bowcock ${ }^{54}$, C. Bozzi ${ }^{16,42}$, S. Braun ${ }^{12}$, M. Brodski ${ }^{42}$, J. Brodzicka ${ }^{29}$, A. Brossa Gonzalo ${ }^{50}$, D. Brundu ${ }^{22}$, E. Buchanan ${ }^{48}$, A. Buonaura ${ }^{44}$, C. Burr ${ }^{56}$, A. Bursche ${ }^{22}$, J. Buytaert ${ }^{42}$, W. Byczynski ${ }^{2}$, S. Cadeddu ${ }^{22}$, H. Cai ${ }^{64}$, R. Calabrese ${ }^{16, g}$, R. Calladine ${ }^{47}$, M. Calvi ${ }^{20, i}$, M. Calvo Gomez ${ }^{40, m}$, A. Camboni ${ }^{40, m}$, P. Campana ${ }^{18}$, D. H. Campora Perez ${ }^{42}$, L. Capriotti ${ }^{56}$, A. Carbone ${ }^{15, e}$, G. Carboni ${ }^{25}$, R. Cardinale ${ }^{19, h}$, A. Cardini ${ }^{22}$, P. Carniti ${ }^{20, i}$, L. Carson ${ }^{52}$, K. Carvalho Akiba ${ }^{2}$, G. Casse ${ }^{54}$, L. Cassina ${ }^{20}$, 
M. Cattaneo ${ }^{42}$, G. Cavallero ${ }^{19, h}$, R. Cenci ${ }^{24, p}$, D. Chamont ${ }^{7}, \quad$ M. G. Chapman ${ }^{48}$, M. Charles ${ }^{8}$, Ph. Charpentier ${ }^{42}$, G. Chatzikonstantinidis ${ }^{47}$, M. Chefdeville ${ }^{4}$, V. Chekalina ${ }^{37}$, C. Chen ${ }^{3}$, S. Chen ${ }^{22}$, S.-G. Chitic ${ }^{42}$, V. Chobanova ${ }^{41}$, M. Chrzaszcz ${ }^{42}$, A. Chubykin ${ }^{33}$, P. Ciambrone ${ }^{18}$, X. Cid Vidal ${ }^{41}$ ，G. Ciezarek $^{42}$, P. E. L. Clarke ${ }^{52}$, M. Clemencic ${ }^{42}$, H. V. Cliff ${ }^{49}$, J. Closier $^{42}$, V. Coco $^{42}$, J. A. B. Coelho ${ }^{7}$, J. Cogan 6 , E. Cogneras ${ }^{5}$, L. Cojocariu ${ }^{32}$, P. Collins ${ }^{42}$, T. Colombo ${ }^{42}$, A. Comerma-Montells ${ }^{12}$, A. Contu ${ }^{22}$, G. Coombs ${ }^{42}$, S. Coquereau ${ }^{40}, \quad$ G. Corti ${ }^{42}, \quad$ M. Corvo ${ }^{16, g}$, C. M. Costa Sobral ${ }^{50}$, B. Couturier ${ }^{42}$, G. A. Cowann $^{52}$, D. C. Craik ${ }^{58}$, A. Crocombe ${ }^{50}$, M. Cruz Torres ${ }^{1}$, R. Currie ${ }^{52}$, C. D’Ambrosio ${ }^{42}$, F. Da Cunha Marinho ${ }^{2}$, C. L. Da Silva ${ }^{74}$, E. Dall'Occo ${ }^{27}$, J. Dalseno ${ }^{48}$, A. Danilina ${ }^{34}$, A. Davis ${ }^{3}$, O. De Aguiar Francisco ${ }^{42}$, K. De Bruyn $^{42}$, S. De Capua ${ }^{56}$, M. De Cian ${ }^{43}$, J. M. De Miranda ${ }^{1}$,L. De Paula ${ }^{2}$, M. De Serio ${ }^{14, d}$, P. De Simone ${ }^{18}$, C.T. Dean ${ }^{53}$, D. Decamp ${ }^{4}$, L. Del Buono ${ }^{8}$, B. Delaney ${ }^{49}$, H.-P. Dembinski ${ }^{11}$, M. Demmer ${ }^{10}$, A. Dendek ${ }^{30}$, D. Derkach ${ }^{37}$, O. Deschamps ${ }^{5}$, F. Desse ${ }^{7}$, F. Dettori ${ }^{54}$, B. Dey ${ }^{65}$, A. Di Canto ${ }^{42}$, P. Di Nezza ${ }^{18}$, S. Didenko ${ }^{70}$, H. Dijkstra ${ }^{42}$, F. Dordei ${ }^{42}$, M. Dorigo ${ }^{42, y}$, A. Dosil Suárez ${ }^{41}$, L. Douglas ${ }^{53}$, A. Dovbnya ${ }^{45}$, K. Dreimanis ${ }^{54}$, L. Dufour ${ }^{27}$, G. Dujany ${ }^{8}$, P. Durante ${ }^{42}$, J. M. Durham ${ }^{74}$, D. Dutta ${ }^{56}$, R. Dzhelyadin ${ }^{39}$, M. Dziewiecki ${ }^{12}$, A. Dziurda ${ }^{29}$, A. Dzyuba ${ }^{33}$, S. Easo ${ }^{51}$, U. Egede ${ }^{55}$, V. Egorychev ${ }^{34}$, S. Eidelman ${ }^{38, w}$, S. Eisenhardt ${ }^{52}$, U. Eitschberger ${ }^{10}$, R. Ekelhof ${ }^{10}$, L. Eklund ${ }^{53}$, S. Ely ${ }^{61}$, A. Ene ${ }^{32}$, S. Escher ${ }^{9}$, S. Esen ${ }^{27}$, T. Evans ${ }^{59}$, A. Falabella ${ }^{15}$, N. Farley ${ }^{47}$, S. Farry ${ }^{54}$, D. Fazzini ${ }^{20,42, i}$, L. Federici ${ }^{25}$, P. Fernandez Declara ${ }^{42}$, A. Fernandez Prieto ${ }^{41}$, F. Ferrari ${ }^{15}$, L. Ferreira Lopes ${ }^{43}$, F. Ferreira Rodrigues ${ }^{2}$, M. Ferro-Luzzi ${ }^{42}$, S. Filippov ${ }^{36}$, R. A. Fini ${ }^{14}$, M. Fiorini ${ }^{16, g}$, M. Firlej ${ }^{30}$, C. Fitzpatrick ${ }^{43}$, T. Fiutowski ${ }^{30}$, F. Fleuret ${ }^{7, b}$, M. Fontana ${ }^{22,42}$, F. Fontanelli ${ }^{19, h}$, R. Forty ${ }^{42}$, V. Franco Lima ${ }^{54}$, M. Frank ${ }^{42}$, C. Frei ${ }^{42}$, J. Fu ${ }^{21, q}$, W. Funk ${ }^{42}$, C. Färber ${ }^{42}$, M. Féo Pereira Rivello Carvalho ${ }^{27}$, E. Gabriel ${ }^{52}$, A. Gallas Torreira ${ }^{41}$, D. Galli ${ }^{15, e}$, S. Gallorini ${ }^{23}$, S. Gambetta ${ }^{52}$, Y. Gan $^{3}$, M. Gandelman ${ }^{2}$, P. Gandini' ${ }^{21}$, Y. Gao ${ }^{3}$, L. M. Garcia Martin ${ }^{72}$, B. Garcia Plana ${ }^{41}$, J. García Pardiñas ${ }^{44}$, J. Garra Tico ${ }^{49}$, L. Garrido ${ }^{40}$, D. Gascon ${ }^{40}$, C. Gaspar ${ }^{42}$, L. Gavardi ${ }^{10}$, G. Gazzoni ${ }^{5}$, D. Gerick ${ }^{12}$, E. Gersabeck ${ }^{56}$, M. Gersabeck ${ }^{56}$, T. Gershon ${ }^{50}$, D. Gerstel ${ }^{6}$, Ph. Ghez ${ }^{4}$, S. Gianì ${ }^{43}$, V. Gibson ${ }^{49}$, O. G. Girard ${ }^{43}$, L. Giubega ${ }^{32}$, K. Gizdov ${ }^{52}$, V. V. Gligorov ${ }^{8}$, D. Golubkov ${ }^{34}$, A. Golutvin ${ }^{55,70}$, A. Gomes ${ }^{1, a}$, I. V. Gorelov ${ }^{35}$, C. Gotti ${ }^{20, i}$, E. Govorkova ${ }^{27}$, J. P. Grabowski ${ }^{12}$, R. Graciani Diaz ${ }^{40}$, L. A. Granado Cardoso ${ }^{42}$, E. Graugés ${ }^{40}$, E. Graverini ${ }^{44}$, G. Graziani ${ }^{17}$, A. Grecu ${ }^{32}$, R. Greim ${ }^{27}$, P. Griffith ${ }^{22}$, L. Grillo ${ }^{56}$, L. Gruber ${ }^{42}$, B. R. Gruberg Cazon ${ }^{57}$, O. Grünberg ${ }^{67}$, C. Gu ${ }^{3}$, E. Gushchin ${ }^{36}$, Yu. Guz ${ }^{39,42}$, T. Gys ${ }^{42}$, C. Göbel ${ }^{62}$, T. Hadavizadeh ${ }^{57}$, C. Hadjivasiliou ${ }^{5}$, G. Haefelii ${ }^{43}$, C. Haen ${ }^{42}$, S. C. Haines ${ }^{49}$, B. Hamilton ${ }^{60}$, X. Han ${ }^{12}$, T. H. Hancock ${ }^{57}$ ， S. Hansmann-Menzemer ${ }^{12}$ ， N. Harnew ${ }^{57 ， \text { S. T. Harnew }}{ }^{48}$ ， T. Harrison ${ }^{54}$, C. Hasse ${ }^{42}$, M. Hatch ${ }^{42}$, J. He ${ }^{63}$, M. Hecker ${ }^{55}$, K. Heinicke ${ }^{10}$, A. Heister ${ }^{10}$, K. Hennessy ${ }^{54}$, L. Henry ${ }^{72}$, E. van Herwijnen ${ }^{42}$, M. He ${ }^{67}$, A. Hicheur ${ }^{2}$, R. Hidalgo Charman ${ }^{56}$, D. Hill ${ }^{57}$, M. Hilton ${ }^{56}$, P. H. Hopchev ${ }^{43}$, W. Hu ${ }^{65}$, W. Huang ${ }^{63}$, Z. C. Huard ${ }^{59}$,

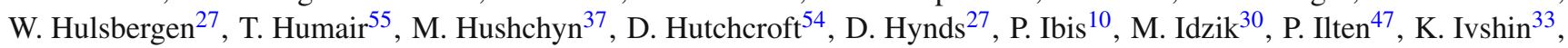
R. Jacobsson ${ }^{42}$, J. Jalocha ${ }^{57}$, E. Jans ${ }^{27}$, A. Jawahery ${ }^{60}$, F. Jiang ${ }^{3}$, M. John ${ }^{57}$, D. Johnson ${ }^{42}$, C. R. Jones ${ }^{49}$, C. Joram ${ }^{42}$, B. Jost ${ }^{42}$, N. Jurik ${ }^{57}$, S. Kandybei ${ }^{45}$, M. Karacson ${ }^{42}$, J. M. Kariuki ${ }^{48}$, S. Karodia ${ }^{53}$, N. Kazeev ${ }^{37}$, M. Kecke ${ }^{12}$, F. Keizer ${ }^{49}$, M. Kelsey ${ }^{61}$, M. Kenzie ${ }^{49}$, T. Ketel ${ }^{28}$, E. Khairullin ${ }^{37}$, B. Khanji ${ }^{42}$, C. Khurewathanakul $\oplus^{43}$, K. E. Kim ${ }^{61}$, T. Kirn ${ }^{9}$, S. Klaver ${ }^{18}$, K. Klimaszewski ${ }^{31}$, T. Klimkovich ${ }^{11}$, S. Koliiev ${ }^{46}$, M. Kolpin ${ }^{12}$, R. Kopecna ${ }^{12}$, P. Koppenburg ${ }^{27}$, I. Kostiuk ${ }^{27}$, S. Kotriakhova ${ }^{33}$, M. Kozeiha ${ }^{5}$, L. Kravchuk ${ }^{36}$, M. Kreps ${ }^{50}$, F. Kress ${ }^{55}$, P. Krokovny ${ }^{38, w}$, W. Krupa ${ }^{30}$, W. Krzemien ${ }^{31}$, W. Kucewicz ${ }^{29,1}$, M. Kucharczyk ${ }^{29}$, V. Kudryavtsev ${ }^{38, w}$, A. K. Kuonen ${ }^{43}$, T. Kvaratskheliya ${ }^{34,42}$, D. Lacarrere ${ }^{42}$, G. Lafferty ${ }^{56}$, A. Lai $^{22}$, D. Lancierini ${ }^{44}$, G. Lanfranchi ${ }^{18}$, C. Langenbruch ${ }^{9}$, T. Latham ${ }^{50}$, C. Lazzeroni ${ }^{47}$, R. Le Gac ${ }^{6}$, A. Leflat ${ }^{35}$, J. Lefrançois ${ }^{7}$, R. Lefèvre ${ }^{5}$, F. Lemaitre ${ }^{42}$, O. Leroy ${ }^{6}$, T. Lesiak ${ }^{29}$, B. Leverington ${ }^{12}$, P.-R. Li ${ }^{63}$, T. Li $^{3}$, Z. Li ${ }^{61}$,

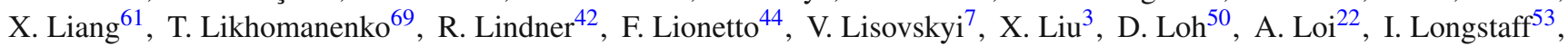
J. H. Lopes ${ }^{2}$, G. H. Lovell ${ }^{49}$, D. Lucchesi ${ }^{23, o}$, M. Lucio Martinez ${ }^{41}$, A. Lupato ${ }^{23}$, E. Luppi 16,g, O. Lupton ${ }^{42}$, A. Lusiani ${ }^{24}$, X. Lyu ${ }^{63}$, F. Machefert ${ }^{7}$, F. Maciuc ${ }^{32}$, V. Macko ${ }^{43}$, P. Mackowiak ${ }^{10}$, S. Maddrell-Mander ${ }^{48}$, O. Maev ${ }^{33,42}$, K. Maguire ${ }^{56}$,

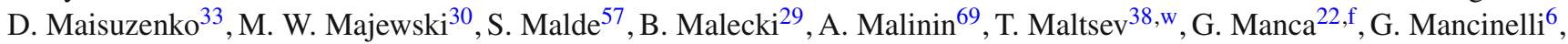
D. Marangotto ${ }^{21, q}$ ， J. Maratas ${ }^{5, v}$ ， J. F. Marchand ${ }^{4}$, U. Marconi ${ }^{15}$, C. Marin Benito ${ }^{7}$, M. Marinangeli ${ }^{43}$, P. Marino ${ }^{43}$, J. Marks ${ }^{12}$ ，P. J. Marshall ${ }^{54}$, G. Martellotti ${ }^{26}$, M. Martin ${ }^{6}$, M. Martinelli ${ }^{42}$, D. Martinez Santos ${ }^{41}$, F. Martinez Vidal ${ }^{72}$, A. Massafferri ${ }^{1}$, M. Materok ${ }^{9}$, R. Matev $^{42}$, A. Mathad ${ }^{50}$, Z. Mathe ${ }^{42}$, C. Matteuzzi ${ }^{20}$, A. Mauri ${ }^{44}$, E. Maurice ${ }^{7, b}$, B. Maurin ${ }^{43}$, A. Mazurov ${ }^{47}$, M. McCann ${ }^{42,55}$, A. McNab56 ${ }^{56}$ R. McNulty ${ }^{13}$, J. V. Mead ${ }^{54}$, B. Meadows ${ }^{59}$, C. Meaux ${ }^{6}$, F. Meier ${ }^{10}$, N. Meinert ${ }^{67}$, D. Melnychuk ${ }^{31}$, M. Merk ${ }^{27}$, A. Merli ${ }^{21, q}$, E. Michielin ${ }^{23}$, D. A. Milanes ${ }^{66}$, E. Millard ${ }^{50}$, M.-N. Minard ${ }^{4}$, L. Minzoni ${ }^{16, g}$, D. S. Mitzel ${ }^{12}$, A. Mogini ${ }^{8}$, J. Molina Rodriguez ${ }^{1, z}$, T. Mombächer ${ }^{10}$, I. A. Monroy ${ }^{66}$, S. Monteil ${ }^{5}$, M. Morandin ${ }^{23}$, G. Morello ${ }^{18}$, M. J. Morello ${ }^{24, t}$, O. Morgunova ${ }^{69}$, J. Moron ${ }^{30}$, A. B. Morris ${ }^{6}$, R. Mountain ${ }^{61}$, F. Muheim ${ }^{52}$, M. Mulder ${ }^{27}$, C. H. Murphy ${ }^{57}$, D. Murray ${ }^{56}$, A. Mödden ${ }^{10}$, D. Müller ${ }^{42}$, J. Müller ${ }^{10}$, K. Müller ${ }^{44}$, V. Müller ${ }^{10}$, P. Naik ${ }^{48}$, T. Nakada ${ }^{43}$, R. Nandakumar ${ }^{51}$, A. Nandi ${ }^{57}$, T. Nanut ${ }^{43}$, I. Nasteva ${ }^{2}$, M. Needham ${ }^{52}$, N. Neri ${ }^{21}$, S. Neubert ${ }^{12}$, N. Neufeld ${ }^{42}$, M. Neuner ${ }^{12}$, T. D. Nguyen ${ }^{43}$, C. Nguyen-Mau ${ }^{43, n}$, S. Nieswand ${ }^{9}$, R. Niet ${ }^{10}$, N. Nikitin ${ }^{35}$, A. Nogay $^{69}$, N. S. Nolte ${ }^{42}$, D. P. O’Hanlon ${ }^{15}$, A. Oblakowska-Mucha ${ }^{30}$, V. Obraztsov ${ }^{39}$, S. Ogilvy ${ }^{18}$, R. Oldeman ${ }^{22, f}$, C. J. G. Onderwater ${ }^{68}$, A. Ossowska ${ }^{29}$, J. M. Otalora Goicochea ${ }^{2}$, P. Owen ${ }^{44}$, A. Oyanguren ${ }^{72}$, P. R. Pais ${ }^{43}$, T. Pajero ${ }^{24, t}$, 
A. Palano ${ }^{14}$ ， M. Palutan ${ }^{18,42}$, G. Panshin ${ }^{71}$ ，A. Papanestis ${ }^{51}$ ， M. Pappagallo5 ${ }^{52}$ L. L. Pappalardo ${ }^{16, g}$, W. Parker ${ }^{60}$, C. Parkes $^{56}$, G. Passaleva ${ }^{17,42}$, A. Pastore ${ }^{14}$, M. Patel $^{55}$, C. Patrignani ${ }^{15, \mathrm{e}}$, A. Pearce ${ }^{42}$, A. Pellegrino ${ }^{27}$, G. Penso ${ }^{26}$, M. Pepe Altarelli ${ }^{42}$, S. Perazzini ${ }^{42}$, D. Pereima ${ }^{34}$, P. Perret ${ }^{5}$, L. Pescatore ${ }^{43}$, K. Petridis ${ }^{48}$, A. Petrolini ${ }^{19, h}$, A. Petrov ${ }^{69}$, S. Petrucci ${ }^{52}$, M. Petruzzo ${ }^{21, q}$, B. Pietrzyk ${ }^{4}$, G. Pietrzyk ${ }^{43}$, M. Pikies $^{29}$, M. Pili ${ }^{57}$, D. Pinci ${ }^{26}$, J. Pinzino ${ }^{42}$, F. Pisani ${ }^{42}$, A. Piucci ${ }^{12}$, V. Placinta ${ }^{32}$, S. Playfer ${ }^{52}$, J. Plews ${ }^{47}$, M. Plo Casasus ${ }^{41}$, F. Polci ${ }^{8}$, M. Poli Lener ${ }^{18}$, A. Poluektov ${ }^{50}$, N. Polukhina ${ }^{70, c}$, I. Polyakov ${ }^{61}$, E. Polycarpo ${ }^{2}$, G. J. Pomery ${ }^{48}$, S. Ponce ${ }^{42}$, A. Popov ${ }^{39}$, D. Popov ${ }^{11,47}$, S. Poslavskii ${ }^{39}$, C. Potterat ${ }^{2}$, E. Price ${ }^{48}$, J. Prisciandaro ${ }^{41}$, C. Prouve ${ }^{48}$, V. Pugatch ${ }^{46}$, A. Puig Navarro ${ }^{44}$, H. Pullen ${ }^{57}$, G. Punzi ${ }^{24, p}$, W. Qian ${ }^{63}$, J. Qin ${ }^{63}$, R. Quagliani ${ }^{8}$, B. Quintana ${ }^{5}$, B. Rachwal ${ }^{30}$, J. H. Rademacker ${ }^{48}$, M. Rama ${ }^{24}$, M. Ramos Pernas ${ }^{41}$, M. S. Rangel ${ }^{2}$, F. Ratnikov ${ }^{37, x}$, G. Raven ${ }^{28}$, M. Ravonel Salzgeber ${ }^{42}$, M. Reboud ${ }^{4}$, F. Redi ${ }^{43}$, S. Reichert ${ }^{10}$, A. C. dos Reis ${ }^{1}$, F. Reiss ${ }^{8}$, C. Remon Alepuz ${ }^{72}$, Z. Ren ${ }^{3}$, V. Renaudin ${ }^{7}$, S. Ricciardi ${ }^{51}$, S. Richards ${ }^{48}$, K. Rinnert ${ }^{54}$, P. Robbe ${ }^{7}$, A. Robert ${ }^{8}$, A. B. Rodrigues ${ }^{43}$, E. Rodrigues ${ }^{59}$, J. A. Rodriguez Lopez $^{66}$, M. Roehrken $^{42}$, S. Roiser ${ }^{42}$, A. Rollings ${ }^{57}$, V. Romanovskiy ${ }^{39}$, A. Romero Vidal ${ }^{41}$, M. Rotondo ${ }^{18}$, M. S. Rudolph ${ }^{61}$, T. Ruf ${ }^{42}$, J. Ruiz Vidal ${ }^{72}$, J. J. Saborido Silva ${ }^{41}$, N. Sagidova ${ }^{33}$,

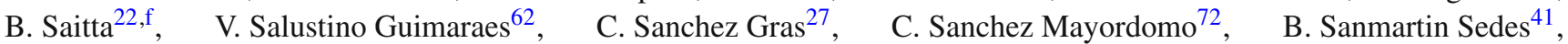
R. Santacesaria ${ }^{26}$, C. Santamarina Rios ${ }^{41}$, M. Santimaria ${ }^{18}$, E. Santovetti ${ }^{25, j}$, G. Sarpis ${ }^{56}$, A. Sarti ${ }^{18, k}$, C. Satriano ${ }^{26, s}$, A. Satta ${ }^{25}$, M. Saur ${ }^{63}$, D. Savrina ${ }^{34,35}$, S. Schael ${ }^{9}$, M. Schellenberg ${ }^{10}$, M. Schiller ${ }^{53}$, H. Schindler ${ }^{42}$, M. Schmelling ${ }^{11}$, T. Schmelzer ${ }^{10}$, B. Schmidt ${ }^{42}$, O. Schneider ${ }^{43}$, A. Schopper ${ }^{42}$, H. F. Schreiner ${ }^{59}$, M. Schubiger ${ }^{43}$, M. H. Schune ${ }^{7}$, R. Schwemmer ${ }^{42}$, B. Sciascia ${ }^{18}$, A. Sciubba ${ }^{26, k}$, A. Semennikov ${ }^{34}$, E. S. Sepulveda ${ }^{8}$, A. Sergi ${ }^{42,47}$, N. Serra ${ }^{44}$, J. Serrano ${ }^{6}$, L. Sestini ${ }^{23}$, A. Seuthe ${ }^{10}$, P. Seyfert ${ }^{42}$, M. Shapkin ${ }^{39}$, Y. Shcheglov ${ }^{33 \dagger}$, T. Shears ${ }^{54}$, L. Shekhtman ${ }^{38, w}$, V. Shevchenko ${ }^{69}$, E. Shmanin ${ }^{70}$, B. G. Siddi ${ }^{16}$, R. Silva Coutinho ${ }^{44}$, L. Silva de Oliveira ${ }^{2}$, G. Simi ${ }^{23, o}$, S. Simone ${ }^{14, d}$, N. Skidmore ${ }^{12}$, T. Skwarnicki ${ }^{61}$, M. W. Slater ${ }^{47}$, J. G. Smeaton ${ }^{49}$, E. Smith ${ }^{9}$, I. T. Smith ${ }^{52}$, M. Smith ${ }^{55}$, M. Soares ${ }^{15}$, 1. Soares Lavra ${ }^{1}$, M. D. Sokoloff ${ }^{59}$, F. J. P. Soler ${ }^{53}$, B. Souza De Paula ${ }^{2}$, B. Spaan ${ }^{10}$, E. Spadaro Norella ${ }^{21, q}$, P. Spradlin ${ }^{53}$, F. Stagni ${ }^{42}$, M. Stahl ${ }^{12}$, S. Stahl ${ }^{42}$, P. Stefko ${ }^{43}$, S. Stefkova ${ }^{55}$, O. Steinkamp ${ }^{44}$, S. Stemmle ${ }^{12}$, O. Stenyakin ${ }^{39}$, M. Stepanova ${ }^{33}$, H. Stevens ${ }^{10}$, A. Stocchi ${ }^{7}$, S. Stone ${ }^{61}$, B. Storaci ${ }^{44}$, S. Stracka ${ }^{24}$, M. E. Stramaglia ${ }^{43}$, M. Straticiuc ${ }^{32}$, U. Straumann ${ }^{44}$, S. Strokov ${ }^{71}$, J. Sun ${ }^{3}$, L. Sun ${ }^{64}$, K. Swientek ${ }^{30}$, T. Szumlak ${ }^{30}$, M. Szymanski ${ }^{63}$, S. T’Jampens ${ }^{4}$, Z. Tang ${ }^{3}$, A. Tayduganov ${ }^{6}$, T. Tekampe ${ }^{10}$, G. Tellarini ${ }^{16}$, F. Teubert ${ }^{42}$, E. Thomas ${ }^{42}$, J. van Tilburg ${ }^{27}$, M. J. Tilley ${ }^{55}$, V. Tisserand ${ }^{5}$, M. Tobin ${ }^{30}$, S. Tolk ${ }^{42}$, L. Tomassetti ${ }^{16, g}$, D. Tonelli ${ }^{24}, \quad$ D. Y. Tou ${ }^{8}, \quad$ R. Tourinho Jadallah Aoude ${ }^{1}, \quad$ E. Tournefier ${ }^{4}, \quad$ M. Traill ${ }^{53}$, M. T. Tran ${ }^{43}$, A. Trisovic ${ }^{49}$, A. Tsaregorodtsev ${ }^{6}$, G Tuci $^{24, p}$, A. Tully ${ }^{49}$, N. Tuning ${ }^{27,42}$, A. Ukleja ${ }^{31}$, A. Usachov ${ }^{7}$, A. Ustyuzhanin ${ }^{37}$, U. Uwer ${ }^{12}$, A. Vagner ${ }^{71}$, V. Vagnoni ${ }^{15}$, A. Valassi ${ }^{42}$, S. Valat ${ }^{42}$, G. Valenti ${ }^{15}$, R. Vazquez Gomez ${ }^{42}$, P. Vazquez Regueiro ${ }^{41}$, S. Vecchi ${ }^{16}$, M. van Veghel ${ }^{27}$, J. J. Velthuis ${ }^{48}$, M. Veltri ${ }^{17, r}$, G. Veneziano ${ }^{57}$, A. Venkateswaran ${ }^{61}$, T. A. Verlage ${ }^{9}$, M. Vernet ${ }^{5}$, M. Veronesi ${ }^{27}$, N. V. Veronika ${ }^{13}$, M. Vesterinen ${ }^{57}$, J. V. Viana Barbosa ${ }^{42}$, D. Vieira ${ }^{63}$, M. Vieites Diaz ${ }^{41}$, H. Viemann ${ }^{67}$, X. Vilasis-Cardona ${ }^{40, m}$, A. Vitkovskiy ${ }^{27}$, M. Vitti ${ }^{49}$, V. Volkov ${ }^{35}$, A. Vollhardt ${ }^{44}$, D. Vom Bruch ${ }^{8}$, B. Voneki ${ }^{42}$, A. Vorobyev ${ }^{33}$, V. Vorobyev ${ }^{38, w}$, J. A. de Vries ${ }^{27}$, C. Vázquez Sierra ${ }^{27}$, R. Waldi ${ }^{67}$, J. Walsh ${ }^{24}$, J. Wang ${ }^{61}$, M. Wang ${ }^{3}$, Y. Wang ${ }^{65}$, Z. Wang ${ }^{44}$, D. R. Ward ${ }^{49}$, H. M. Wark ${ }^{54}$, N. K. Watson ${ }^{47}$, D. Websdale ${ }^{55}$, A. Weiden ${ }^{44}$, C. Weisser ${ }^{58}$, M. Whitehead ${ }^{9}$, J. Wicht $^{50}$, G. Wilkinson ${ }^{57}$, M. Wilkinson ${ }^{61}$, I. Williams ${ }^{49}$, M. R. J. Williams ${ }^{56}$, M. Williams ${ }^{58}$, T. Williams ${ }^{47}$, F. F. Wilson ${ }^{42,51}$, J. Wimberley ${ }^{60}$, M. Winn ${ }^{7}$, J. Wishahi ${ }^{10}$, W. Wislicki ${ }^{31}$, M. Witek ${ }^{29}$, G. Wormser ${ }^{7}$, S. A. Wotton ${ }^{49}$, K. Wyllie ${ }^{42}$, D. Xiao ${ }^{65}$, Y. Xie ${ }^{65}$, A. Xu ${ }^{3}, \quad$ M. Xu ${ }^{65}, \quad$ Q. Xu ${ }^{63}$, Z. Xu ${ }^{3}$ Z. Xu ${ }^{4}$, Z. Yang ${ }^{3}$, Z. Yang ${ }^{60}$, Y. Yao ${ }^{61}$, L. E. Yeomans ${ }^{54}$, H. Yin ${ }^{65}$, J. Yu ${ }^{65, a b}$, X. Yuan ${ }^{61}$, O. Yushchenko ${ }^{39}$, K. A. Zarebski ${ }^{47}$, M. Zavertyaev ${ }^{11, \mathrm{c}}$, D. Zhang ${ }^{65}$, L. Zhang ${ }^{3}$, W. C. Zhang ${ }^{3, \text { aa }}$, Y. Zhang ${ }^{7}$, A. Zhelezov ${ }^{12}$, Y. Zheng ${ }^{63}$, X. Zhu ${ }^{3}$, V. Zhukov ${ }^{9,35}$, J. B. Zonneveld ${ }^{52}$, S. Zucchelli ${ }^{15}$

\footnotetext{
${ }^{1}$ Centro Brasileiro de Pesquisas Físicas (CBPF), Rio de Janeiro, Brazil

${ }^{2}$ Universidade Federal do Rio de Janeiro (UFRJ), Rio de Janeiro, Brazil

${ }^{3}$ Center for High Energy Physics, Tsinghua University, Beijing, China

${ }^{4}$ Univ. Grenoble Alpes, Univ. Savoie Mont-Blanc, CNRS/IN2P3-LAPP, Annecy, France

${ }^{5}$ Clermont Université, Université Blaise Pascal, CNRS/IN2P3, LPC, Clermont-Ferrand, France

${ }^{6}$ Aix-Marseille Univ, CNRS/IN2P3, CPPM, Marseille, France

${ }^{7}$ LAL, Univ. Paris-Sud, CNRS/IN2P3, Université Paris-Saclay, Orsay, France

${ }^{8}$ LPNHE, Sorbonne Université, Paris Diderot, Sorbonne Paris Cité, CNRS/IN2P3, Paris, France

${ }^{9}$ I. Physikalisches Institut, RWTH Aachen University, Aachen, Germany

${ }^{10}$ Fakultät Physik, Technische Universität Dortmund, Dortmund, Germany

${ }^{11}$ Max-Planck-Institut für Kernphysik (MPIK), Heidelberg, Germany

${ }^{12}$ Physikalisches Institut, Ruprecht-Karls-Universität Heidelberg, Heidelberg, Germany

${ }^{13}$ School of Physics, University College Dublin, Dublin, Ireland
} 
${ }^{14}$ INFN Sezione di Bari, Bari, Italy

${ }^{15}$ INFN Sezione di Bologna, Bologna, Italy

${ }^{16}$ INFN Sezione di Ferrara, Ferrara, Italy

${ }^{17}$ INFN Sezione di Firenze, Florence, Italy

${ }^{18}$ INFN Laboratori Nazionali di Frascati, Frascati, Italy

${ }^{19}$ INFN Sezione di Genova, Genoa, Italy

${ }^{20}$ INFN, Sezione di Milano-Bicocca, Milan, Italy

${ }^{21}$ INFN Sezione di Milano, Milan, Italy

22 INFN Sezione di Cagliari, Monserrato, Italy

${ }^{23}$ INFN Sezione di Padova, Padua, Italy

${ }^{24}$ INFN Sezione di Pisa, Pisa, Italy

${ }^{25}$ INFN Sezione di Roma Tor Vergata, Rome, Italy

${ }^{26}$ INFN Sezione di Roma La Sapienza, Rome, Italy

${ }^{27}$ Nikhef National Institute for Subatomic Physics, Amsterdam, The Netherlands

${ }^{28}$ Nikhef National Institute for Subatomic Physics and VU University Amsterdam, Amsterdam, The Netherlands

${ }^{29}$ Henryk Niewodniczanski Institute of Nuclear Physics Polish Academy of Sciences, Kraków, Poland

${ }^{30}$ Faculty of Physics and Applied Computer Science, AGH-University of Science and Technology, Kraków, Poland

${ }^{31}$ National Center for Nuclear Research (NCBJ), Warsaw, Poland

${ }^{32}$ Horia Hulubei National Institute of Physics and Nuclear Engineering, Bucharest-Magurele, Romania

${ }^{33}$ Petersburg Nuclear Physics Institute (PNPI), Gatchina, Russia

${ }^{34}$ Institute of Theoretical and Experimental Physics (ITEP), Moscow, Russia

${ }^{35}$ Institute of Nuclear Physics, Moscow State University (SINP MSU), Moscow, Russia

${ }^{36}$ Institute for Nuclear Research of the Russian Academy of Sciences (INR RAS), Moscow, Russia

${ }^{37}$ Yandex School of Data Analysis, Moscow, Russia

${ }^{38}$ Budker Institute of Nuclear Physics (SB RAS), Novosibirsk, Russia

${ }^{39}$ Institute for High Energy Physics (IHEP), Protvino, Russia

${ }^{40}$ ICCUB, Universitat de Barcelona, Barcelona, Spain

${ }^{41}$ Instituto Galego de Física de Altas Enerxías (IGFAE), Universidade de Santiago de Compostela, Santiago de Compostela, Spain

${ }^{42}$ European Organization for Nuclear Research (CERN), Geneva, Switzerland

${ }^{43}$ Institute of Physics, Ecole Polytechnique Fédérale de Lausanne (EPFL), Lausanne, Switzerland

${ }^{44}$ Physik-Institut, Universität Zürich, Zurich, Switzerland

${ }^{45}$ NSC Kharkiv Institute of Physics and Technology (NSC KIPT), Kharkiv, Ukraine

${ }^{46}$ Institute for Nuclear Research of the National Academy of Sciences (KINR), Kyiv, Ukraine

${ }^{47}$ University of Birmingham, Birmingham, UK

${ }^{48}$ H.H. Wills Physics Laboratory, University of Bristol, Bristol, UK

${ }^{49}$ Cavendish Laboratory, University of Cambridge, Cambridge, UK

${ }^{50}$ Department of Physics, University of Warwick, Coventry, UK

${ }^{51}$ STFC Rutherford Appleton Laboratory, Didcot, UK

${ }^{52}$ School of Physics and Astronomy, University of Edinburgh, Edinburgh, UK

${ }^{53}$ School of Physics and Astronomy, University of Glasgow, Glasgow, UK

${ }^{54}$ Oliver Lodge Laboratory, University of Liverpool, Liverpool, UK

${ }^{55}$ Imperial College London, London, UK

${ }^{56}$ School of Physics and Astronomy, University of Manchester, Manchester, UK

${ }^{57}$ Department of Physics, University of Oxford, Oxford, UK

${ }^{58}$ Massachusetts Institute of Technology, Cambridge, MA, USA

${ }^{59}$ University of Cincinnati, Cincinnati, OH, USA

${ }^{60}$ University of Maryland, College Park, MD, USA

${ }^{61}$ Syracuse University, Syracuse, NY, USA

62 Pontifícia Universidade Católica do Rio de Janeiro (PUC-Rio), Rio de Janeiro, Brazil, associated to ${ }^{2}$

${ }^{63}$ University of Chinese Academy of Sciences, Beijing, China, associated to ${ }^{3}$

${ }^{64}$ School of Physics and Technology, Wuhan University, Wuhan, China, associated to ${ }^{3}$

${ }^{65}$ Institute of Particle Physics, Central China Normal University, Wuhan, Hubei, China, associated to ${ }^{3}$ 
${ }^{66}$ Departamento de Fisica, Universidad Nacional de Colombia, Bogotá, Colombia, associated to ${ }^{8}$

${ }^{67}$ Institut für Physik, Universität Rostock, Rostock, Germany, associated to ${ }^{12}$

${ }^{68}$ Van Swinderen Institute, University of Groningen, Groningen, The Netherlands, associated to ${ }^{27}$

${ }^{69}$ National Research Centre Kurchatov Institute, Moscow, Russia, associated to ${ }^{34}$

${ }^{70}$ National University of Science and Technology "MISIS", Moscow, Russia, associated to ${ }^{34}$

${ }^{71}$ National Research Tomsk Polytechnic University, Tomsk, Russia, associated to ${ }^{34}$

${ }^{72}$ Instituto de Fisica Corpuscular, Centro Mixto Universidad de Valencia-CSIC, Valencia, Spain, associated to ${ }^{40}$

${ }^{73}$ University of Michigan, Ann Arbor, USA associated to ${ }^{61}$

${ }^{74}$ Los Alamos National Laboratory (LANL), Los Alamos, USA associated to ${ }^{61}$

${ }^{a}$ Universidade Federal do Triângulo Mineiro (UFTM), Uberaba, MG, Brazil

${ }^{\mathrm{b}}$ Laboratoire Leprince-Ringuet, Palaiseau, France

${ }^{c}$ P.N. Lebedev Physical Institute, Russian Academy of Science (LPI RAS), Moscow, Russia

${ }^{\mathrm{d}}$ Università di Bari, Bari, Italy

${ }^{\mathrm{e}}$ Università di Bologna, Bologna, Italy

${ }^{\mathrm{f}}$ Università di Cagliari, Cagliari, Italy

g Università di Ferrara, Ferrara, Italy

${ }^{\mathrm{h}}$ Università di Genova, Genoa, Italy

${ }^{i}$ Università di Milano Bicocca, Milan, Italy

${ }^{\mathrm{j}}$ Università di Roma Tor Vergata, Rome, Italy

${ }^{\mathrm{k}}$ Università di Roma La Sapienza, Rome, Italy

${ }^{1}$ AGH-University of Science and Technology, Faculty of Computer Science, Electronics and Telecommunications, Kraków, Poland

${ }^{\mathrm{m}}$ LIFAELS, La Salle, Universitat Ramon Llull, Barcelona, Spain

${ }^{\mathrm{n}}$ Hanoi University of Science, Hanoi, Vietnam

${ }^{\circ}$ Università di Padova, Padua, Italy

p Università di Pisa, Pisa, Italy

${ }^{q}$ Università degli Studi di Milano, Milan, Italy

${ }^{\mathrm{r}}$ Università di Urbino, Urbino, Italy

${ }^{\mathrm{s}}$ Università della Basilicata, Potenza, Italy

${ }^{\mathrm{t}}$ Scuola Normale Superiore, Pisa, Italy

u Università di Modena e Reggio Emilia, Modena, Italy

${ }^{v}$ MSU, Iligan Institute of Technology (MSU-IIT), Iligan, Philippines

${ }^{\mathrm{w}}$ Novosibirsk State University, Novosibirsk, Russia

${ }^{x}$ National Research University Higher School of Economics, Moscow, Russia

${ }^{y}$ Sezione INFN di Trieste, Trieste, Italy

${ }^{\mathrm{z}}$ Escuela Agrícola Panamericana, San Antonio de Oriente, Honduras

${ }^{\text {aa }}$ School of Physics and Information Technology, Shaanxi Normal University (SNNU), Xi' an, China

${ }^{a b}$ Physics and Micro Electronic College, Hunan University, Changsha, China

${ }^{\dagger}$ Deceased 